\title{
Classical stability of a homogeneous, anisotropic inflating space-time
}

\author{
Timothy R. Dulaney, ${ }^{*}$ Moira I. Gresham, ${ }^{\dagger}$ and Mark B. Wise ${ }^{\ddagger}$ \\ California Institute of Technology, Pasadena, California 91125, USA
}

(Received 6 February 2008; published 17 April 2008)

\begin{abstract}
We study the classical stability of an anisotropic space-time seeded by a spacelike, fixed-norm, dynamical vector field in a vacuum-energy-dominated inflationary era. It serves as a model for breaking isotropy during the inflationary era. We find that, for a range of parameters, the linear differential equations for small perturbations about the background do not have a growing mode. We also examine the energy of fluctuations about this background in flat space. If the kinetic terms for the vector field do not take the form of a field strength tensor squared, then there is a negative energy mode and the background is unstable. For the case where the kinetic term is of the form of a field strength tensor squared, we show that classical solutions to the equations of motion that are close to the background solution have energy greater than or equal to zero. This suggests that the background is metastable.
\end{abstract}

DOI: 10.1103/PhysRevD.77.083510

PACS numbers: $98.80 . \mathrm{Cq}, 04.20 .-\mathrm{q}, 04.40 . \mathrm{Nr}$

\section{INTRODUCTION}

Inflation has become the standard paradigm for very early Universe cosmology. It supposes an era when the Universe was dominated by vacuum energy. During this era any classical inhomogeneities are smoothed out by the exponential expansion of the Universe. The inhomogeneities that we observe today originate in small quantum fluctuations that have their wavelength redshifted outside the horizon by the exponential expansion of the Universe. At some time, the Universe exits the inflationary era and transitions to a radiation-dominated era and then eventually to a matter-dominated era. After inflation, the Universe expands slower than the speed at which light travels. This allows the perturbations with wavelengths that are outside of the horizon to eventually reenter the horizon and generate the inhomogeneities we observe in the microwave background (as well as the large scale structure of the Universe).

Since we have no direct probes of the inflationary era, we are compelled to consider the possibility that some tenets of physics - tenets that are fundamental to our current understanding of the Universe - only reflect postinflationary developments. One such tenet is that rotational invariance is not spontaneously (or explicitly) broken. This symmetry implies angular momentum conservation and is necessary for the classification of elementary particles by their spin (i.e., their angular momentum in their rest frame).

Recently the possibility that rotational invariance is broken during the inflationary era has been studied [1-6]. In Ref. [1] it was noted that if the breaking of rotational invariance is small there is a very simple and unique signature on the spectrum of density perturbations and hence on the anisotropy of the microwave background

\footnotetext{
*dulaney@theory.caltech.edu

moira@theory.caltech.edu

wise@ theory.caltech.edu
}

radiation. Ackerman et al. also introduced a simple model for breaking rotational invariance during the inflationary era. They calculated the influence of the breaking of rotational invariance on the spectrum of density perturbations, verifying the expectations based on their general arguments. The Lagrange density for the model has the usual Einstein-Hilbert action, a cosmological constant term with vacuum energy density $\rho_{\Lambda}$ and the following terms containing the four-vector field $u^{\sigma}$,

$$
\begin{aligned}
\mathcal{L}_{u}= & -\beta_{1} \nabla^{\mu} u^{\sigma} \nabla_{\mu} u_{\sigma}-\beta_{2}\left(\nabla_{\mu} u^{\mu}\right)^{2}-\beta_{3} \nabla^{\mu} u^{\sigma} \nabla_{\sigma} u_{\mu} \\
& +\lambda\left(u^{\mu} u_{\mu}-m^{2}\right) .
\end{aligned}
$$

Here $\lambda$ is a Lagrange multiplier that enforces the constraint $g_{\mu \nu} u^{\mu} u^{\nu}=m^{2}$. We take $m^{2}>0$ so the four-vector $u^{\sigma}$ is spacelike.

This model has a homogeneous but anisotropic background solution to the classical equations of motion. We choose the $x_{3}$ axis to be aligned along the four-vector field,

$$
u^{0}=0, \quad u^{1}=u^{2}=0, \quad \text { and } \quad u^{3}=\frac{m}{b(t)},
$$

resulting in a space-time metric of the form

$$
\mathrm{d} s^{2}=-\mathrm{d} t^{2}+a(t)^{2} \mathrm{~d} x_{\perp}^{2}+b(t)^{2} \mathrm{~d} x_{3}^{2} .
$$

The breaking of rotational invariance by the four-vector field causes the $x_{3}$ direction to expand at a different rate than the $x_{1}$ and $x_{2}$ directions. Explicitly, ${ }^{1}$

$$
a(t)=e^{H_{a} t}, \quad b(t)=e^{H_{b} t},
$$

where the Hubble parameters are related to the vacuum energy, the scale of rotational invariance breaking, and parameters in the vector Lagrangian by the following equations,

\footnotetext{
${ }^{1}$ It is assumed that the dynamics immediately preceding the inflationary era was rotationally invariant so that $a(0)=b(0)$.
} 


$$
\begin{aligned}
& H_{a}=\frac{\dot{a}}{a}=H_{b}\left(1+16 \pi G \beta_{1} m^{2}\right), \\
& H_{b}=\frac{\dot{b}}{b}=\sqrt{\frac{8 \pi G \rho_{\Lambda}}{\left(1+8 \pi G \beta_{1} m^{2}\right)\left(3+32 \pi G \beta_{1} m^{2}\right)} .}
\end{aligned}
$$

Notice that the background solution does not depend on the parameters $\beta_{2,3}$, and as $m \rightarrow 0$ the rate of expansion is the same for all spatial dimensions.

The purpose of this paper is to study the classical stability of this solution. We first consider the linear differential equations that result from expanding the classical equations of motion to linear order about the background solution discussed above. We find that, for small $G \beta_{i} m^{2}$, small perturbations do not grow provided that $\beta_{1}>0$ and $\beta_{1}+\beta_{2}+\beta_{3} \geq 0$. Next we consider the energy of these solutions in flat space. Negative energy modes indicate an instability that might not show up in the analysis of the linearized equations of motion.

A special role is played by the case $\beta_{1}+\beta_{2}+\beta_{3}=0$ where, in flat space, the kinetic terms for the vector field take the form of a field strength tensor squared. In this case, we find that when the energy density is evaluated to quadratic order in fluctuations about the background, all propagating modes have positive energy. However, when that equality is not satisfied, there is a mode that propagates with negative energy. For the case where the kinetic term is of the form of a field strength tensor squared, we show that classical solutions to the Euler-Lagrange equations of motion in flat space that are close to the background solution have energy greater than or equal to zero.

Models with vector fields that spontaneously break Lorentz symmetry were first introduced in [7]. Similar fixed-norm, timelike, Lorentz-violating vector field models have been extensively studied; constraints required by theoretical and observational consistency have been placed on parameters in this theory [7-19]. For a review, see [20].

Others have classified gauge invariant perturbations in anisotropic scenarios [21,22]; however, we choose to work in a particular (nonstandard) gauge for calculational convenience.

\section{STRATEGY}

The effect of an isotropy-breaking vector field on the expansion of the Universe during inflation was recently studied [1]. In this model, the four-vector $u^{\mu}$ is nonzero only during the time interval $0<t<t_{*}$, where $t=0$ is the beginning of inflation and $t_{*}$ is the end of inflation. We assume that the dynamics is rotationally invariant during reheating and thereafter. During the time interval $0<t<$ $t_{*}$, the dynamics of interest in this paper is governed by the action

$$
S=\int \mathrm{d}^{4} x \sqrt{-g}\left(\frac{1}{16 \pi G} R-\rho_{\Lambda}+\mathcal{L}_{u}\right),
$$

where $\mathcal{L}_{u}$ is given in Eq. (1). The homogeneous background inflationary space-time solution is given in Eqs. (3)-(5). [9]

The energy-momentum tensor for $u^{\mu}$ derived from (1) is

$$
\begin{aligned}
T_{\mu \nu}^{(u)}= & 2 \beta_{1}\left(\nabla_{\mu} u^{\rho} \nabla_{\nu} u_{\rho}-\nabla^{\rho} u_{\mu} \nabla_{\rho} u_{\nu}\right)-2\left[\nabla_{\rho}\left(u_{(\mu} J^{\rho}{ }_{\nu)}\right)\right. \\
& \left.+\nabla_{\rho}\left(u^{\rho} J_{(\mu \nu)}\right)-\nabla_{\rho}\left(u_{(\mu} J_{\nu)}^{\rho}\right)\right] \\
& +2 m^{-2} u_{\sigma} \nabla_{\rho} J^{\rho \sigma} u_{\mu} u_{\nu}+g_{\mu \nu} \mathcal{L}_{u}
\end{aligned}
$$

where $J^{\mu}{ }_{\sigma}$ is the current tensor,

$$
J_{\sigma}^{\mu}=-\beta_{1} \nabla^{\mu} u_{\sigma}-\beta_{2} \delta_{\sigma}^{\mu} \nabla_{\rho} u^{\rho}-\beta_{3} \nabla_{\sigma} u^{\mu} .
$$

Given Eqs. (2) and (7), the nonvanishing components of the background stress tensor are

$$
\begin{gathered}
T_{00}^{(u)}=\beta_{1} m^{2}\left(\frac{\dot{b}}{b}\right)^{2}, \quad T_{11}^{(u)}=T_{22}^{(u)}=\beta_{1} m^{2} a^{2}\left(\frac{\dot{b}}{b}\right)^{2}, \\
T_{33}^{(u)}=\beta_{1} m^{2}\left(\dot{b}^{2}-2 \ddot{b} b-4 \frac{\dot{a} \dot{b} b}{a}\right) .
\end{gathered}
$$

The components of the energy-momentum tensor in our chosen background are independent of $\beta_{2}$ and $\beta_{3}$. For a nontrivial vector contribution to the stress tensor (and consequently for an anisotropic metric resulting from the field equations), one must require that $\beta_{1} \neq 0$. Note that the background solution does not satisfy the weak energy condition. An inflating background solution requires

$$
8 \pi G m^{2} \beta_{1}>-\frac{1}{2} \text {. }
$$

This is not a strong bound on $\beta_{1}$ since we are interested in backgrounds that have a small violation of isotropy, i.e., $G m^{2} \ll 1$.

In this paper we study the classical stability of the homogeneous background solution to the equations of motion. We expand the equations of motion to linear order about the homogeneous background. Since the background is homogeneous it is convenient to Fourier transform the small fluctuations about the background in the comoving spatial coordinates $\left(x_{1}, x_{2}, x_{3}\right)$ and examine the time dependence that the linearized equations of motion imply. The physical wave vectors $\bar{k}_{i}, i=1,2$, and $\bar{k}_{3}$ are related to the comoving wave vectors by $\bar{k}_{i}=k_{i} / a(t)$ and $\bar{k}_{3}=$ $k_{3} / b(t)$.

We begin with a flat-space stability analysis neglecting gravity since, when $\vec{k} \gg H$, such an analysis captures the essential physics. Then we perform a stability analysis including gravity in two regions, $\vec{k} \gg H$ and $\vec{k} \ll H$. We do not treat the transition region where the physical wavelengths of the modes cross the horizon. In the final section of this paper we examine the flat-space energy of solutions to the equations of motion. Negative energy solutions indicate an instability that might not be evident from a study of the linearized equations of motion. 


\section{STABILITY ANALYSIS NEGLECTING GRAVITY}

In the short-wavelength limit, $\vec{k} \gg H$, it is physically intuitive that modes will not be able to resolve space-time curvature. Therefore we should be able to derive dispersion relations for small fluctuations in $u_{\mu}$ about a flat background space-time that will receive corrections suppressed by $\mathrm{Gm}^{2}$ when gravity is included. In this case, we have the action

$$
\begin{aligned}
S= & \int d^{4} x\left(-\beta_{1}\left(\partial^{\mu} u^{\sigma}\right)\left(\partial_{\mu} u_{\sigma}\right)-\beta_{2}\left(\partial_{\mu} u^{\mu}\right)^{2}\right. \\
& \left.-\beta_{3}\left(\partial^{\mu} u^{\sigma}\right)\left(\partial_{\sigma} u_{\mu}\right)+\lambda\left(u^{\mu} u_{\mu}-m^{2}\right)\right) .
\end{aligned}
$$

The Euler-Lagrange equation for $\lambda$ gives

$$
\eta_{\mu \nu} u^{\mu} u^{\nu}=m^{2}
$$

while the equation of motion for the vector field gives

$$
\lambda u^{\mu}+\beta_{1} \partial_{\nu} \partial^{\nu} u^{\mu}+\left(\beta_{2}+\beta_{3}\right) \partial^{\mu}\left(\partial_{\nu} u^{\nu}\right)=0 .
$$

We can use Eq. (11) to solve for $\lambda$ by taking the inner product of the vector Euler-Lagrange equation (12) with the vector,

$$
\lambda=-\frac{\beta_{1}}{m^{2}} u_{\mu} \partial_{\nu} \partial^{\nu} u^{\mu}-\left(\frac{\beta_{2}+\beta_{3}}{m^{2}}\right) u_{\mu} \partial^{\mu}\left(\partial_{\nu} u^{\nu}\right) .
$$

We consider small perturbations about the background, $\bar{u}^{\mu}=m \eta^{\mu 3}, \bar{\lambda}=0$, and $\bar{g}_{\mu \nu}=\eta_{\mu \nu}$. We denote small perturbations about the background vector field by $v^{\mu}$ so that

$$
u^{\mu}=\bar{u}^{\mu}+v^{\mu} .
$$

Since we are neglecting gravity, all Greek indices are raised and lowered by the Minkowski metric. The perturbation about the background value of the Lagrange multiplier, $\delta \lambda=\lambda-\bar{\lambda}$, is a function of the vector field perturbations through Eq. (13),

$$
m \delta \lambda+\left(\beta_{2}+\beta_{3}\right) \partial_{3}\left(\partial_{\nu} v^{\nu}\right)=0 .
$$

From Eq. (11) we see that $v_{3}=0$. And by expanding Eq. (12) to first order in perturbations, we have the following equations for the nontrivial vector field perturbations $(i \in\{1,2\})$ :

$$
\begin{gathered}
\beta_{1} \partial_{\nu} \partial^{\nu} v^{0}+\left(\beta_{2}+\beta_{3}\right) \partial^{0}\left(\partial_{\nu} v^{\nu}\right)=0, \\
\beta_{1} \partial_{\nu} \partial^{\nu} v^{i}+\left(\beta_{2}+\beta_{3}\right) \partial^{i}\left(\partial_{\nu} v^{\nu}\right)=0 .
\end{gathered}
$$

We Fourier transform the components of $v^{\mu}$, and in terms of the Fourier modes (transformed in both space and time),

$$
\begin{aligned}
& v^{0}(\omega, \vec{k})=k_{3} \theta_{1}(\omega, \vec{k}) \quad \text { and } \\
& v^{i}(\omega, \vec{k})=k_{i} \theta_{2}(\omega, \vec{k})+\epsilon_{i j} k_{j} \psi(\omega, \vec{k}),
\end{aligned}
$$

where $i, j \in\{1,2\}$, and we have arranged for all scalar $(\theta)$ and pseudoscalar ${ }^{2}(\psi)$ components to have the same mass dimension. With this decomposition, linear combinations of the Fourier transform of Eqs. (15) become

$$
\begin{gathered}
{\left[\beta_{1}\left(k_{\perp}^{2}+k_{3}^{2}\right)-\left(\beta_{1}+\beta_{2}+\beta_{3}\right) \omega^{2}\right] k_{3} \theta_{1}} \\
+\left(\beta_{2}+\beta_{3}\right) \omega k_{\perp}^{2} \theta_{2}=0, \\
{\left[\beta_{1}\left(k_{\perp}^{2}+k_{3}^{2}-\omega^{2}\right)+\left(\beta_{2}+\beta_{3}\right) k_{\perp}^{2}\right] \theta_{2}} \\
-\left(\beta_{2}+\beta_{3}\right) \omega k_{3} \theta_{1}=0, \\
\beta_{1} k_{\perp}^{2}\left(k_{\perp}^{2}+k_{3}^{2}-\omega^{2}\right) \psi=0,
\end{gathered}
$$

where $k_{\perp}^{2} \equiv \sum_{i=1}^{2} k_{i} k_{i}$.

We first consider the case where $\beta_{1}+\beta_{2}+\beta_{3} \neq 0$. The scalar mode $\theta_{1}=\left(k_{\perp}^{2} / \omega k_{3}\right) \theta_{2}$ propagates with the dispersion relation

$$
\omega^{2}=k_{\perp}^{2}+k_{3}^{2} .
$$

The pseudoscalar mode also propagates with this dispersion relation. The scalar mode $\theta_{1}=\left(\omega / k_{3}\right) \theta_{2}$ propagates with the dispersion relation

$$
\omega^{2}=k_{\perp}^{2}+k_{3}^{2}\left(\frac{\beta_{1}}{\beta_{1}+\beta_{2}+\beta_{3}}\right) .
$$

When $\beta_{1}+\beta_{2}+\beta_{3}=0$, Eq. (17) becomes a constraint equation and therefore the number of scalar modes is decreased by 1 while the pseudoscalar equation remains dynamical. A mode disappears at first order when $\beta_{1}+$ $\beta_{2}+\beta_{3}=0$ because there is an enhanced gauge symmetry. At first order in perturbations, the transformation $v_{\mu} \rightarrow$ $v_{\mu}+\partial_{\mu} \alpha\left(t, x_{1}, x_{2}\right)$ leaves the Lagrangian invariant. The scalar function $\alpha$ is restricted to have no $x_{3}$ dependence at first order by the constraint $u_{\mu} u^{\mu}=m^{2}$. The disappearing mode,

$$
v^{0}(\omega, \vec{k})=\omega \theta_{2}(\omega, \vec{k}), \quad v^{i}(\omega, \vec{k})=k^{i} \theta_{2}(\omega, \vec{k}),
$$

satisfies (in coordinate space) $v_{\mu}(t, \vec{x})=\partial_{\mu} \phi(t, \vec{x})$ for $\mu=0,1,2$. When $\beta_{2}+\beta_{3}=-\beta_{1}$, Eqs. (15) imply that $\phi(t, \vec{x})$ is independent of $x_{3}$. Thus the mode (22) becomes a gauge artifact when $\beta_{1}+\beta_{2}+\beta_{3}=0$.

\section{STABILITY ANALYSIS INCLUDING GRAVITY}

In this analysis we have four-vector field fluctuations and ten metric fluctuations to consider. However, we can use diffeomorphism invariance to remove some of them. Under an infinitesimal coordinate transformation, $x^{\mu} \rightarrow$ $x^{\mu}+\xi^{\mu}$, the vector field transforms to $u^{\lambda}+\Delta u^{\lambda}$, where

$$
\Delta u^{\lambda}=u^{\mu} \partial_{\mu} \xi^{\lambda}-\xi^{\mu} \partial_{\mu} u^{\lambda},
$$

\footnotetext{
${ }^{2}$ We use the name "pseudoscalar" for the $\psi$ 's not because these objects are odd under parity but in order to distinguish them from the $\theta$ 's. The two different kinds of scalars decouple.
} 
while the metric transforms to $g_{\mu \nu}+\Delta g_{\mu \nu}$, where

$$
\Delta g_{\mu \nu}=-\left(\nabla_{\mu} \xi_{\nu}+\nabla_{\nu} \xi_{\mu}\right)
$$

We choose a gauge where the first order fluctuations in the (contravariant) four-vector field about their background, $\bar{u}^{\mu}=\eta^{\mu 3} m / b(t)$, vanish [i.e. $\delta\left(u^{\mu}\right)=v^{\mu}=0$ where $\left.u^{\mu}=\bar{u}^{\mu}+v^{\mu}\right]$. This gauge condition is satisfied by fixing

$$
\begin{aligned}
-v^{\lambda} & =\left.\Delta u^{\lambda}\right|_{1^{\mathrm{st}} \text { order }}=\bar{u}^{\mu} \partial_{\mu} \xi^{\lambda}-\xi^{\mu} \partial_{\mu} \bar{u}^{\lambda} \\
& =\frac{m}{b(t)} \partial_{3} \xi^{\lambda}+\delta_{3}^{\lambda} \xi^{0} \frac{m}{b(t)} H_{b} .
\end{aligned}
$$

It is important to note that the gauge freedom has not been completely exploited. The condition $\delta\left(u^{\mu}\right)=0$ is invariant under gauge transformations of the form $x^{\mu} \rightarrow$ $x^{\mu}+\zeta^{\mu}$, where

$$
\begin{aligned}
\zeta^{\mu}= & \left(f_{0}\left(t, x_{1}, x_{2}\right), f_{1}\left(t, x_{1}, x_{2}\right), f_{2}\left(t, x_{1}, x_{2}\right), f_{3}\left(t, x_{1}, x_{2}\right)\right. \\
& \left.-H_{b} x_{3} f_{0}\left(t, x_{1}, x_{2}\right)\right) .
\end{aligned}
$$

Once the above four functions of three coordinate variables are chosen, the gauge has been completely specified. This residual gauge freedom will be used in the analysis of longwavelength fluctuations.

The vector field satisfies the Lagrange multiplier equation, $u^{2}=m^{2}$. This equation and our choice of gauge imply that

$$
\left(\delta g_{\mu \nu}\right) u^{\mu} u^{\nu}+2 g_{\mu \nu}\left(v^{\mu}\right) u^{\nu}=\left(\delta g_{33}\right) \frac{m^{2}}{b^{2}}=0,
$$

and thus we are left with nine metric fluctuations to consider.

Without loss of generality, we have chosen coordinates such that the background vector field lies entirely along the $x^{3}$ direction. Since isotropy is broken, only an $\mathrm{SO}(2)$ spatial symmetry remains. Let the Roman indices $i, j, k$ be $\mathrm{SO}(2)$ indices that run from 1 to 2 . Define the "comoving " metric perturbations, $h_{\mu \nu}$, through the following equation,

$$
\begin{aligned}
d s^{2}= & -\left(1+h_{00}\right) d t^{2}+2 a(t) h_{0 i} d t d x^{i}+2 b(t) h_{03} d t d x^{3} \\
& +2 a(t) b(t) h_{i 3} d x^{i} d x^{3}+a(t)^{2}\left(\delta_{i j}+h_{i j}\right) d x^{i} d x^{j} \\
& +b(t)^{2}\left(d x^{3}\right)^{2}
\end{aligned}
$$

We work to first order in the small perturbations. After making our gauge choice, there are nine perturbations, as well as nine independent equations for the system. One can show that the equations of motion for the vector field are equivalent to the energy-momentum conservation equations. Furthermore, energy-momentum conservation (and thus the equations of motion) follow from Einstein's equa- tions. The identity to first order in perturbations is

$$
8 \pi G \delta\left(\nabla_{\mu} T_{\nu}^{\mu}\right)=\nabla^{\mu} \delta E_{\mu \nu},
$$

where

$$
\delta E_{\mu \nu}=\delta\left(G_{\mu \nu}\right)-8 \pi G \delta\left(T_{\mu \nu}\right) .
$$

Finally, only nine of Einstein's equations are independent. The interdependence among Einstein's equations in our gauge can be found by working out the identity above for $\nu=3$, since the left-hand side vanishes identically,

$$
\left(\partial_{t}+2\left(H_{a}+H_{b}\right)\right) \frac{\delta E_{03}}{b(t)}=\delta^{i j} \frac{\partial_{i} \delta E_{j 3}}{a(t)^{2} b(t)}+\frac{\partial_{3} \delta E_{33}}{b(t)^{3}} .
$$

Thus we take, as our complete set of equations at first order in small perturbations, all of Einstein's equations except for $\delta E_{33}$.

We Fourier transform

$$
h_{\mu \nu}\left(t, k_{1}, k_{2}, k_{3}\right)=\int \frac{d^{3} x}{(2 \pi)^{3 / 2}} h_{\mu \nu}\left(t, x^{1}, x^{2}, x^{3}\right) e^{-i \vec{k} \cdot \vec{x}},
$$

and define

$$
\bar{k}_{i}=k_{i} / a(t), \quad \bar{k}_{\perp}^{2}=\bar{k}_{i} \bar{k}_{i}, \quad \text { and } \quad \bar{k}_{3}=k_{3} / b(t),
$$

where $i \in\{1,2\}$ and summation is implied over repeated indices. Also, define the constants

$$
\lambda_{n}=8 \pi G m^{2} \beta_{n} \quad \text { and } \quad \Lambda=8 \pi G \rho_{\Lambda} .
$$

Then the Fourier-transformed field equations become

$$
\begin{aligned}
\delta E_{00}= & \left(\left(\Lambda+\bar{k}_{3}^{2}\left(\lambda_{1}+\lambda_{2}+\lambda_{3}\right)\right) h_{00}+2 i \bar{k}_{3}\left(\lambda_{1} H_{b}-H_{a}\right) h_{03}\right. \\
& -i\left(H_{a}+H_{b}\right) \bar{k}_{i} h_{0 i}-\bar{k}_{3} \bar{k}_{i} h_{i 3}+\frac{1}{2}\left(\bar{k}_{\perp}^{2}+\bar{k}_{3}^{2}\left(1-2 \lambda_{2}\right)\right. \\
& \left.+\left(H_{a}+H_{b}\right) \partial_{t}\right) h_{i i}-\frac{1}{2} \bar{k}_{i} \bar{k}_{j} h_{i j}=0,
\end{aligned}
$$

$$
\begin{aligned}
\frac{2 \delta E_{0 i}}{a(t)}= & -i \bar{k}_{i}\left(H_{a}+H_{b}\right) h_{00}-\bar{k}_{3} \bar{k}_{i} h_{03}+\left(2 \Lambda-2 H_{a}^{2}\right. \\
& -2 H_{a} H_{b}-2 H_{b}^{2}\left(1+\lambda_{1}\right)+\bar{k}_{\perp}^{2}+\bar{k}_{3}^{2}\left(1+2 \lambda_{1}\right. \\
& \left.\left.+2 \lambda_{3}\right)\right) h_{0 i}-\bar{k}_{i} \bar{k}_{j} h_{0 j}+i \bar{k}_{3}\left(-H_{a}+H_{b}\left(1+4 \lambda_{1}\right)\right. \\
& \left.+\partial_{t}\right) h_{i 3}+i\left(\bar{k}_{j} \partial_{t} h_{i j}-\bar{k}_{i} \partial_{t} h_{j j}\right)=0,
\end{aligned}
$$

$$
\begin{aligned}
\frac{\delta E_{03}}{b(t)}= & i \bar{k}_{3}\left(\lambda_{1} H_{b}-H_{a}\right) h_{00}+\left(\Lambda-3 H_{a}^{2}+\lambda_{1}\left(4 H_{a} H_{b}\right.\right. \\
& \left.\left.+H_{b}^{2}+2 \bar{k}_{3}^{2}\right)+\frac{1}{2}\left(1+2 \lambda_{1}-2 \lambda_{3}\right) \bar{k}_{\perp}^{2}\right) h_{03} \\
& -\frac{1}{2} \bar{k}_{3} \bar{k}_{i} h_{0 i}+i \bar{k}_{i} \frac{1}{2}\left(1+2 \lambda_{1}-2 \lambda_{3}\right)\left(H_{a}-H_{b}+\partial_{t}\right) h_{i 3} \\
& -i \bar{k}_{3} \frac{1}{2}\left(H_{a}-H_{b}\left(1+2 \lambda_{1}\right)+\partial_{t}\right) h_{i i}=0
\end{aligned}
$$




$$
\begin{aligned}
\frac{\delta E_{i j}}{a(t)^{2}}= & \delta_{i j}\left(-\left(H_{a}^{2}+H_{a} H_{b}+\left(1+\lambda_{1}\right) H_{b}^{2}\right)+\frac{1}{2} \bar{k}_{\perp}^{2}+\frac{1}{2}\left(1-2 \lambda_{2}\right) \bar{k}_{3}^{2}-\frac{1}{2}\left(H_{a}+H_{b}\right) \partial_{t}\right) h_{00}-\frac{1}{2} \bar{k}_{i} \bar{k}_{j} h_{00} \\
+ & i \bar{k}_{3} \delta_{i j}\left(H_{a}+\left(1+2 \lambda_{1}\right) H_{b}+\partial_{t}\right) h_{03}-i \bar{k}_{(i}\left(H_{a}+H_{b}+\partial_{t}\right) h_{j) 0}+i \delta_{i j} \bar{k}_{l}\left(H_{a}+H_{b}+\partial_{t}\right) h_{0 l} \\
+ & \bar{k}_{3}\left(\delta_{i j} \bar{k}_{l} h_{l 3}-\bar{k}_{(i} h_{j) 3}\right)-\frac{1}{2} \delta_{i j}\left(\bar{k}_{3}^{2}\left(1-2 \lambda_{2}\right)+\left(2 H_{a}+H_{b}+\partial_{t}\right) \partial_{t}\right) h_{k k} \\
+ & \left(\Lambda-\left(H_{a}^{2}+H_{a} H_{b}+H_{b}^{2}\left(1+\lambda_{1}\right)\right)+\frac{1}{2} \bar{k}_{3}^{2}\left(1+2 \lambda_{1}+2 \lambda_{3}\right)+\frac{1}{2}\left(2 H_{a}+H_{b}+\partial_{t}\right) \partial_{t}\right) h_{i j}=0, \\
\frac{2 \delta E_{i 3}}{a(t) b(t)}= & -\bar{k}_{3} \bar{k}_{i} h_{00}-i \bar{k}_{i}\left(1+2 \lambda_{1}-2 \lambda_{3}\right)\left(2 H_{b}+\partial_{t}\right) h_{03}-i \bar{k}_{3}\left(3 H_{a}-H_{b}\left(1+4 \lambda_{1}\right)+\partial_{t}\right) h_{0 i} \\
& +\left(2 \Lambda+H_{a}^{2}\left(2 \lambda_{1}-2 \lambda_{3}-5\right)+H_{a} H_{b}\left(1+10 \lambda_{1}-2 \lambda_{3}\right)-2 H_{b}^{2}\left(1+\lambda_{1}-2 \lambda_{3}\right)+4 \bar{k}_{3}^{2} \lambda_{1}\right) h_{i 3} \\
& +\left(1+2 \lambda_{1}-2 \lambda_{3}\right)\left(\delta_{i j}\left(\bar{k}_{\perp}^{2}+\left(2 H_{a}+H_{b}+\partial_{t}\right) \partial_{t}\right)-\bar{k}_{i} \bar{k}_{j}\right) h_{j 3}+\bar{k}_{3}\left(\bar{k}_{i} h_{j j}-\bar{k}_{j} h_{i j}\right)=0 .
\end{aligned}
$$

\section{A. Short-wavelength limit of the field equations}

Here we consider the limit $(H / \bar{k}) \rightarrow 0$, which corresponds to modes with physical wavelengths much shorter than the Hubble radius. Such modes have periods much shorter than the Hubble time; therefore we can treat the $\bar{k}_{i}$ as constants independent of time (i.e. $H_{a, b}$ are effectively zero in this limit). The background solution in Eq. (5) gives $\sqrt{\Lambda} \sim H_{b}$, and thus we also take $\left(\Lambda / \bar{k}^{2}\right) \rightarrow 0$.

The various degrees of freedom in the field equations decouple into six equations governing the scalar modes and three equations governing the pseudoscalar modes if one uses the decomposition $(i, j=1,2)$

$$
\begin{gathered}
h_{00}=\bar{k}_{\perp}^{2} \bar{\Theta}_{1}, \quad h_{0 i}=\bar{k}_{3}\left(\bar{k}_{i} \bar{\Theta}_{2}+\epsilon_{i j} \bar{k}_{j} \bar{\Psi}_{1}\right), \quad h_{03}=\bar{k}_{3}^{2} \bar{\Theta}_{3}, \\
h_{i j}=\delta_{i j} \frac{\bar{k}_{\perp}^{2}}{2} \bar{\Theta}_{4}+\left[\bar{k}_{i} \bar{k}_{j}-\delta_{i j} \frac{\bar{k}_{\perp}^{2}}{2}\right] \bar{\Theta}_{5}+\bar{k}_{(i} \epsilon_{j) k} \bar{k}_{k} \bar{\Psi}_{2}, \\
h_{i 3}=\bar{k}_{3}\left(\bar{k}_{i} \bar{\Theta}_{6}+\epsilon_{i j} \bar{k}_{j} \bar{\Psi}_{3}\right) .
\end{gathered}
$$

Note that all of the scalar $(\bar{\Theta})$ and pseudoscalar $(\bar{\Psi})$ components have the same mass dimension. We make the ansatz for the time dependence of the fields above,

$$
\bar{\Theta}_{l}(t, \vec{k})=\bar{\Theta}_{l}(\omega, \vec{k}) e^{i \omega t} \quad \text { and } \quad \bar{\Psi}_{l}(t, \vec{k})=\bar{\Psi}_{l}(\omega, \vec{k}) e^{i \omega t} .
$$

The decomposition (34) aids in solving the field equations in the usual way: linear combinations of Einstein's equations that resemble the above decomposition lead to equations coupling only (pseudo)scalar fields.

There are six scalar fields in the mode decomposition (34). The functional and algebraic constraints on these fields are given by the field equations:

$$
\begin{aligned}
& 0=\frac{\delta E_{00}}{\bar{k}_{\perp}^{2}}=\bar{k}_{3}^{2}\left(\lambda_{1}+\lambda_{2}+\lambda_{3}\right) \bar{\Theta}_{1}+\left[\frac{\bar{k}_{\perp}^{2}}{4}+\frac{\bar{k}_{3}^{2}}{2}\left(1-2 \lambda_{2}\right)\right] \bar{\Theta}_{4}-\frac{\bar{k}_{\perp}^{2}}{4} \bar{\Theta}_{5}-\bar{k}_{3}^{2} \bar{\Theta}_{6}, \\
& 0=\frac{2 \bar{k}_{i} \delta E_{0 i}}{a(t) \bar{k}_{3} \bar{k}_{\perp}^{2}}=\bar{k}_{3}^{2}\left(1+2 \lambda_{1}+2 \lambda_{3}\right) \bar{\Theta}_{2}-\bar{k}_{3}^{2} \bar{\Theta}_{3}+\frac{\omega}{\bar{k}_{3}}\left(\frac{\bar{k}_{\perp}^{2}}{2} \bar{\Theta}_{4}-\frac{\bar{k}_{\perp}^{2}}{2} \bar{\Theta}_{5}-\bar{k}_{3}^{2} \bar{\Theta}_{6}\right), \\
& 0=\frac{\delta E_{03}}{b(t) \bar{k}_{3}^{2}}=-\frac{\bar{k}_{\perp}^{2}}{2} \bar{\Theta}_{2}+\left[\frac{\bar{k}_{\perp}^{2}}{2}\left(1+2 \lambda_{1}-2 \lambda_{3}\right)+2 \bar{k}_{3}^{2} \lambda_{1}\right] \bar{\Theta}_{3}+\frac{\omega}{\bar{k}_{3}}\left(\frac{\bar{k}_{\perp}^{2}}{2} \bar{\Theta}_{4}-\frac{\bar{k}_{\perp}^{2}}{2}\left(1+2 \lambda_{1}-2 \lambda_{3}\right) \bar{\Theta}_{6}\right), \\
& 0=\frac{\delta E_{i i}}{a(t)^{2} \bar{k}_{\perp}^{2}}=\left[\frac{\bar{k}_{\perp}^{2}}{2}+\bar{k}_{3}^{2}\left(1-2 \lambda_{2}\right)\right] \bar{\Theta}_{1}-\bar{k}_{3} \omega \bar{\Theta}_{2}-2 \frac{\omega \bar{k}_{3}^{3}}{\bar{k}_{\perp}^{2}} \bar{\Theta}_{3}+\left[\frac{\omega^{2}}{2}+\bar{k}_{3}^{2}\left(\lambda_{1}+2 \lambda_{2}+\lambda_{3}-\frac{1}{2}\right)\right] \bar{\Theta}_{4}+\bar{k}_{3}^{2} \bar{\Theta}_{6}, \\
& 0=\frac{\bar{k}_{i} \bar{k}_{j} \delta E_{i j}}{a(t)^{2} \bar{k}_{\perp}^{4}}=\frac{\bar{k}_{3}^{2}}{2}\left(1-2 \lambda_{2}\right) \bar{\Theta}_{1}-\frac{\omega \bar{k}_{3}^{3}}{\bar{k}_{\perp}^{2}} \bar{\Theta}_{3}+\left[\frac{\omega^{2}}{4}-\frac{\bar{k}_{3}^{2}}{4}\left(1-2 \lambda_{1}-4 \lambda_{2}-2 \lambda_{3}\right)\right] \bar{\Theta}_{4}+\left[\frac{\bar{k}_{3}^{2}}{4}\left(1+2 \lambda_{1}+2 \lambda_{3}\right)-\frac{\omega^{2}}{4}\right] \bar{\Theta}_{5}, \\
& 0=\frac{\bar{k}_{i} \delta E_{i 3}}{a(t) b(t) \bar{k}_{3} \bar{k}_{\perp}^{2}}=\frac{\omega \bar{k}_{3}}{2} \bar{\Theta}_{2}-\frac{\bar{k}_{\perp}^{2}}{2} \bar{\Theta}_{1}+\frac{\omega \bar{k}_{3}}{2}\left(1+2 \lambda_{1}-2 \lambda_{3}\right) \bar{\Theta}_{3}+\frac{\bar{k}_{\perp}^{2}}{4}\left(\bar{\Theta}_{4}-\bar{\Theta}_{5}\right)-\left[\frac{\omega^{2}}{2}\left(1+2 \lambda_{1}-2 \lambda_{3}\right)-2 \lambda_{1} \bar{k}_{3}^{2}\right] \bar{\Theta}_{6} .
\end{aligned}
$$

Let us consider the dynamical degrees of freedom. We shall schematically denote $\omega \bar{\Theta}(\omega, \vec{k}) \sim \partial_{t} \bar{\Theta}(t, \vec{k}) \sim \dot{\bar{\Theta}}(t)$. The first equation is a constraint. When $\beta_{1}+\beta_{2}+\beta_{3} \neq 0,{ }^{3}$ it can be solved to give $\bar{\Theta}_{1}(t)=\bar{\Theta}_{1}\left(\bar{\Theta}_{4}(t), \bar{\Theta}_{5}(t), \bar{\Theta}_{6}(t)\right)$. The next

\footnotetext{
${ }^{2}$ Recall the definition $\lambda_{n} \equiv 8 \pi G m^{2} \beta_{n}$.
} 
two equations can be solved to give $\bar{\Theta}_{2 .}(t)=$ $\bar{\Theta}_{2}\left(\dot{\bar{\Theta}}_{4}(t), \dot{\bar{\Theta}}_{5}(t), \dot{\bar{\Theta}}_{6}(t)\right)$ and $\bar{\Theta}_{3}(t)=\bar{\Theta}_{3}\left(\dot{\bar{\Theta}}_{4}(t), \overline{\bar{\Theta}}_{5}(t)\right.$, $\left.\dot{\bar{\Theta}}_{6}(t)\right)$. In the last three equations, $\bar{\Theta}_{2}$ and $\bar{\Theta}_{3}$ appear only as $\dot{\bar{\Theta}}_{2}(t)$ and $\dot{\bar{\Theta}}_{3}(t)$. Thus, after making the substitutions for $\bar{\Theta}_{1}, \bar{\Theta}_{2}$, and $\bar{\Theta}_{3}$, the last three equations form a system of second order ordinary differential equations of three functions. Thus, when $\beta_{1}+\beta_{2}+\beta_{3} \neq 0$, we expect three distinct dynamical modes.

When $\beta_{1}+\beta_{2}+\beta_{3}=0$, the function $\bar{\Theta}_{1}$ drops out of the constraint equation. Then the first equation can be solved for $\bar{\Theta}_{4}\left(\bar{\Theta}_{5}(t), \bar{\Theta}_{6}(t)\right)$, and the next two for $\bar{\Theta}_{2}(t)=$ $\bar{\Theta}_{2}\left(\dot{\bar{\Theta}}_{5}(t), \dot{\bar{\Theta}}_{6}(t)\right)$ and $\bar{\Theta}_{3}(t)=\bar{\Theta}_{3}\left(\dot{\bar{\Theta}}_{5}(t)\right.$, $\left.\dot{\bar{\Theta}}_{6}(t)\right)$. Since $\bar{\Theta}_{1}$ enters the above equations with no time derivatives, one of the last three equations can be used to eliminate $\bar{\Theta}_{1}$, and the remaining two become second order differential equations in time of two functions, $\bar{\Theta}_{5}(t)$ and $\bar{\Theta}_{6}(t)$. Thus, when $\beta_{1}+\beta_{2}+\beta_{3}=0$, we expect only two distinct dynamical modes in the scalar sector.

Here we present the propagating modes and their dispersion relations. The first scalar mode relates the amplitudes of the various fields by

$$
\bar{\Theta}_{1}=\frac{\bar{k}_{3}}{\omega} \bar{\Theta}_{2}=\bar{\Theta}_{4}=\frac{\bar{k}_{\perp}^{2} \bar{\Theta}_{5}}{2 \omega^{2}-\bar{k}_{\perp}^{2}} ; \quad \bar{\Theta}_{3}=0=\bar{\Theta}_{6},
$$

and propagates with the dispersion relation

$$
\omega^{2}=\bar{k}_{\perp}^{2}+\bar{k}_{3}^{2}\left(1+2 \lambda_{1}+2 \lambda_{3}\right) .
$$

The next scalar mode is characterized by the amplitude relationships

$$
\begin{aligned}
\bar{\Theta}_{1} & =\bar{\Theta}_{4}=\bar{\Theta}_{5}=\frac{2 \bar{k}_{3} \omega}{\omega^{2}+\bar{k}_{\perp}^{2}} \bar{\Theta}_{2} \\
& =\frac{2 \bar{k}_{3} \omega}{\bar{k}_{\perp}^{2}\left(1+2 \lambda_{1}+2 \lambda_{3}\right)} \bar{\Theta}_{3}=\frac{2}{1+2 \lambda_{1}+2 \lambda_{3}} \bar{\Theta}_{6},
\end{aligned}
$$

and propagates with the dispersion relation

$$
\omega^{2}=\bar{k}_{\perp}^{2}+\bar{k}_{3}^{2}\left(\frac{\lambda_{1}\left(1+2 \lambda_{1}+2 \lambda_{3}\right)}{\lambda_{1}+\lambda_{1}^{2}-\lambda_{3}^{2}}\right) .
$$

The last propagating scalar mode only occurs when $\beta_{1}+$ $\beta_{2}+\beta_{3} \neq 0$ and is characterized by the amplitude relationship

$$
\begin{aligned}
\bar{\Theta}_{5} & =\frac{\bar{k}_{3}}{\omega} \bar{\Theta}_{2}=\frac{2 \bar{k}_{3}\left(1+\lambda_{1}\right)}{\omega\left(1+2 \lambda_{1}+2 \lambda_{3}\right)} \bar{\Theta}_{3} \\
& =\frac{2\left(1+\lambda_{1}\right)}{1+2 \lambda_{1}+2 \lambda_{3}} \bar{\Theta}_{6}=\frac{\bar{k}_{\perp}^{2}}{\left(\omega^{2}+\frac{\bar{k}_{3}^{2} \lambda_{1}\left(1+2 \lambda_{1}+2 \lambda_{3}\right)}{1+\lambda_{1}}\right)} \bar{\Theta}_{1} \\
& =\frac{\bar{k}_{\perp}^{2}}{\left(\bar{k}_{\perp}^{2}-2 \frac{\bar{k}_{3}^{2} \lambda_{1}\left(1+2 \lambda_{1}+2 \lambda_{3}\right)}{1+\lambda_{1}}\right)} \bar{\Theta}_{4},
\end{aligned}
$$

and the dispersion relation

$$
\begin{aligned}
\omega^{2}= & \bar{k}_{\perp}^{2}+\bar{k}_{3}^{2}\left(\frac{\lambda_{1}\left(1+2 \lambda_{1}+2 \lambda_{3}\right)}{\left(1+\lambda_{1}\right)}\right) \\
& \times\left(\frac{1-\lambda_{1}-3 \lambda_{2}-\lambda_{3}}{\lambda_{1}+\lambda_{2}+\lambda_{3}}\right) .
\end{aligned}
$$

This last mode corresponds to the flat-space mode (22). As we found in the flat-space analysis, this mode is absent when $\beta_{1}+\beta_{2}+\beta_{3}=0$. [Note that, in the shortwavelength limit, $a(t)$ and $b(t)$ are treated as constants, so covariant derivatives become ordinary derivatives and thus $\beta_{1}+\beta_{2}+\beta_{3}=0$ corresponds to the case where the Lagrange density takes the form of a field strength squared - as in the flat-space analysis.]

For general Fourier components, we can guarantee that $\omega^{2} \geq 0$ if and only if the coefficient of the $\bar{k}_{3}^{2}$ term is positive semidefinite. Thus we see that the stability of the modes gives the following conditions on the $\lambda_{n}$ 's [and thus, recalling Eq. (28), the $\beta_{n}$ 's]:

$$
\begin{aligned}
& \lambda_{1}+\lambda_{3} \geq-\frac{1}{2}, \quad \frac{\lambda_{1}\left(1+2 \lambda_{1}+2 \lambda_{3}\right)}{\lambda_{1}+\lambda_{1}^{2}-\lambda_{3}^{2}} \geq 0, \\
& \frac{\lambda_{1}\left(1+2 \lambda_{1}+2 \lambda_{3}\right)}{\left(1+\lambda_{1}\right)}\left(\frac{1-2 \lambda_{2}}{\lambda_{1}+\lambda_{2}+\lambda_{3}}-1\right) \geq 0 .
\end{aligned}
$$

We see that only the last equation gives us a condition on $\beta_{2}$. In the limit that $G m^{2} \ll 1$, we have the following condition,

$$
\frac{\lambda_{1}}{\lambda_{1}+\lambda_{2}+\lambda_{3}}=\frac{\beta_{1}}{\beta_{1}+\beta_{2}+\beta_{3}} \geq 0,
$$

which is identical to what we found in the flat-space analysis. The other two constraints are trivially satisfied in this limit.

The following equations for the pseudoscalar fields follow from the field equations:

$$
\begin{aligned}
& 0=\frac{2 \epsilon_{i j} \delta E_{0 i} \bar{k}_{j}}{\bar{k}_{3} \bar{k}_{\perp}^{2} a(t)}=\left[\bar{k}_{\perp}^{2}+\bar{k}_{3}^{2}\left(1+2 \lambda_{1}+2 \lambda_{3}\right)\right] \bar{\Psi}_{1}-\frac{\omega}{\bar{k}_{3}}\left(\frac{\bar{k}_{\perp}^{2}}{2} \bar{\Psi}_{2}+\bar{k}_{3}^{2} \bar{\Psi}_{3}\right), \\
& 0=\frac{\epsilon_{l j} \bar{k}_{l} \bar{k}_{i} \delta E_{i j}}{\bar{k}_{\perp}^{4} a(t)^{2}}=-\frac{\omega \bar{k}_{3}}{2} \bar{\Psi}_{1}+\frac{\bar{k}_{3}^{2}}{2} \bar{\Psi}_{3}+\left[\frac{\omega^{2}}{4}-\frac{\bar{k}_{3}^{2}}{4}\left(1+2 \lambda_{1}+2 \lambda_{3}\right)\right] \bar{\Psi}_{2}, \\
& 0=\frac{\epsilon_{i j} \delta E_{i 3} \bar{k}_{j}}{\bar{k}_{3} \bar{k}_{\perp}^{2} a(t) b(t)}=\frac{\omega \bar{k}_{3}}{2} \bar{\Psi}_{1}-\frac{\bar{k}_{\perp}^{2}}{4} \bar{\Psi}_{2}-\left[\frac{\omega^{2}-\bar{k}_{\perp}^{2}}{2}\left(1+2 \lambda_{1}-2 \lambda_{3}\right)-2 \lambda_{1} \bar{k}_{3}^{2}\right] \bar{\Psi}_{3},
\end{aligned}
$$


where $\lambda_{n}=8 \pi G m^{2} \beta_{n}$. Here, the first equation is a constraint, and there are two distinct dynamical modes. The pseudoscalar eigenmode $\left(2 \omega \bar{k}_{3} / \bar{k}_{\perp}^{2}\right) \bar{\Psi}_{1}=\bar{\Psi}_{2}, \quad \bar{\Psi}_{3}=0$ propagates with the dispersion relation

$$
\omega^{2}=\bar{k}_{\perp}^{2}+\bar{k}_{3}^{2}\left(1+2 \lambda_{1}+2 \lambda_{3}\right) .
$$

The second pseudoscalar eigenmode is given by $\left(2 \bar{k}_{3} / \omega\right)\left(1+2 \lambda_{1}+2 \lambda_{3}\right) \bar{\Psi}_{1}=\left(1+2 \lambda_{1}+2 \lambda_{3}\right) \bar{\Psi}_{2}=$ $2 \bar{\Psi}_{3}$ and propagates with the dispersion relation

$$
\omega^{2}=\bar{k}_{\perp}^{2}+\bar{k}_{3}^{2}\left(\frac{\lambda_{1}\left(1+2 \lambda_{1}+2 \lambda_{3}\right)}{\lambda_{1}+\lambda_{1}^{2}-\lambda_{3}^{2}}\right) .
$$

Note that the value of $\beta_{2}$ does not affect the stability of any of the propagating pseudoscalar modes. Also, the two pseudoscalar dispersion relations were already found to characterize two of the propagating modes in the scalar sector.

When $\beta_{1}=-\beta_{3}$ and $\beta_{2}=0$ (the Maxwell case), all modes propagate with the speed of light. However, all modes would not necessarily propagate with the speed of light if we only required $\beta_{1}+\beta_{2}+\beta_{3}=0$.

In the limit $G m^{2} \ll 1$ (or equivalently $m^{2} \ll M_{p}^{2}$, where $M_{p}$ is the Planck mass) we have $\lambda_{n} \ll 1$. The flat-space analysis considered earlier is insensitive to $\mathcal{O}\left(\lambda_{n}\right)$ corrections to the dispersion relations and (of course) neglects gravitational degrees of freedom. Here we show that the more general analysis simplifies to the flat-space analysis. Since, in our gauge, $\delta\left(u_{\mu}\right)=\delta g_{\mu \nu} \bar{u}^{\nu}=(\mathrm{m} / \mathrm{b}) \delta g_{\mu 3}$, comparing Eqs. (16) and (34) we expect that

$$
\theta_{1} \propto \bar{\Theta}_{3}, \quad \theta_{2} \propto \bar{\Theta}_{6}, \quad \text { and } \quad \psi \propto \bar{\Psi}_{3} .
$$

In the $\lambda_{n} \ll 1$ limit, the dispersion relation for the scalar mode, (38), and the pseudoscalar mode, (44), has the first order approximation

$$
\omega^{2}=\bar{k}_{\perp}^{2}+\bar{k}_{3}^{2}\left[1+\left(\frac{\left(\lambda_{1}+\lambda_{3}\right)^{2}}{\lambda_{1}}\right)\right],
$$

with amplitude relationship $\left(\bar{k}_{3} \omega / \bar{k}_{\perp}^{2}\right) \bar{\Theta}_{3}=\bar{\Theta}_{6}$. The dispersion relation in Eq. (40) has the first order approximation

$$
\omega^{2}=\bar{k}_{\perp}^{2}+\bar{k}_{3}^{2}\left(\frac{\beta_{1}}{\beta_{1}+\beta_{2}+\beta_{3}}\right)\left[1-3 \lambda_{2}+\lambda_{3}\right],
$$

with amplitude relationship $\left(\bar{k}_{3} / \omega\right) \bar{\Theta}_{3}=\bar{\Theta}_{6}$.

The $\lambda_{n} \rightarrow 0$ limit of dispersion relations (38), (40), and (44) and the corresponding mode amplitude relationships, along with Eq. (45), lead to the propagating modes and dispersion relations found in Sec. III. (In the flat-space analysis, one can rescale the Fourier modes in order to put them in the form of $\bar{k}_{\perp}$ and $\bar{k}_{3}$ since this simply results in an overall constant rescaling of the integration measure.) We found only three (two when $\beta_{1}+\beta_{2}+\beta_{3}=0$ ) distinct modes in the analysis neglecting gravity because the $\bar{\Theta}_{6}=0=\bar{\Theta}_{3}$ and $\bar{\Psi}_{3}=0$ modes are purely gravitational.
The purely gravitational modes have the following exact dispersion relation,

$$
\omega^{2}=k_{\perp}^{2}+k_{3}^{2}\left(1+2 \lambda_{1}+2 \lambda_{3}\right) .
$$

As $\lambda_{n} \rightarrow 0$, the gravitational modes have the usual graviton dispersion relation (as expected). We see that the intuitive flat-space analysis is reproduced by the more general analysis involving coupling to gravity.

In [10], Lim carried out a similar perturbative analysis of a fixed-norm, timelike, Lorentz-violating vector field in a de Sitter background. Lim considers the model of Ref. [9], which is a slight simplification of the model in Ref. [17]. ${ }^{4}$ $\mathrm{He}$ found rescaled mode propagation speeds very similar to those that we found when $\beta_{1}+\beta_{2}+\beta_{3} \neq 0$. There is a one-to-one correspondence of the inverse of his propagation speeds with our $x_{3}$-direction propagation speeds when $m^{2} \rightarrow-m^{2}$.

\section{B. Long-wavelength limit of the field equations}

We now consider the behavior of the modes after they cross the Hubble horizon and work in the limit where $\bar{k} / H \ll 1$. First simplifying Einstein's equations in this limit and then performing a mode decomposition yields simple differential equations for the various modes. The field equations decouple into six equations governing the scalar $(\Theta)$ modes and three equations governing the pseudoscalar $(\Psi)$ modes if one uses the decomposition $(i, j=$ $1,2)$

$$
\begin{gathered}
h_{00}=\Theta_{1}, \quad h_{0 i}=\frac{1}{k_{\perp}}\left(k_{i} \Theta_{2}+\epsilon_{i j} k_{j} \Psi_{1}\right), \quad h_{03}=\Theta_{3}, \\
h_{i j}=\frac{\delta_{i j}}{2} \Theta_{4}+\left[\frac{k_{i} k_{j}}{k_{\perp}^{2}}-\frac{\delta_{i j}}{2}\right] \Theta_{5}+\frac{k_{(i} \epsilon_{j) k} k_{k}}{k_{\perp}^{2}} \Psi_{2}, \\
h_{i 3}=\frac{1}{k_{\perp}}\left(k_{i} \Theta_{6}+\epsilon_{i j} k_{j} \Psi_{3}\right),
\end{gathered}
$$

where $k_{\perp}=\sqrt{k_{1}^{2}+k_{2}^{2}}$. Note that all of the scalar $(\Theta)$ and pseudoscalar $(\Psi)$ components have the same mass dimension and are zeroth order in $k_{\perp}, k_{3}$.

The scalar mode Einstein equations are

$$
\begin{aligned}
0= & H_{b}\left(3+4 \lambda_{1}\right) \Theta_{1}(t)+\partial_{t} \Theta_{4}(t), \\
0= & 4 H_{b}\left(1+\lambda_{1}\right)\left(H_{b}\left(3+4 \lambda_{1}\right)+\partial_{t}\right) \Theta_{1}(t) \\
& +\left(\left(3+4 \lambda_{1}\right) H_{b} \partial_{t}+\partial_{t}^{2}\right) \Theta_{4}(t), \\
0= & \left(H_{b}\left(3+4 \lambda_{1}\right) \partial_{t}+\partial_{t}^{2}\right) \Theta_{5}(t), \\
0= & \left(2 H_{b}^{2} \lambda_{1}\left(3+2 \lambda_{1}\right)+H_{b}\left(3+4 \lambda_{1}\right) \partial_{t}+\partial_{t}^{2}\right) \Theta_{6}(t),
\end{aligned}
$$

where we have used the background solution $H_{a}=$ $H_{b}\left(1+2 \lambda_{1}\right)$ and $\Lambda=H_{b}^{2}\left(1+\lambda_{1}\right)\left(3+4 \lambda_{1}\right)$. The other two scalar equations are trivially zero. This means that $\Theta_{2}(t)$ and $\Theta_{3}(t)$ are unconstrained functions of time. The

${ }^{4}$ The action in [17] includes a term quartic in the vector field. 
solutions for the remaining scalar modes are

$$
\begin{gathered}
\Theta_{1}(t)=\Theta_{1}(0) e^{-t H_{b}\left(3+4 \lambda_{1}\right)}, \\
\Theta_{4}(t)=\Theta_{1}(0)\left(e^{-t H_{b}\left(3+4 \lambda_{1}\right)}-1\right)+\Theta_{4}(0), \\
\Theta_{5}(t)=\Theta_{5}(0) e^{-t H_{b}\left(3+4 \lambda_{1}\right)}+A_{5}\left(e^{-t H_{b}\left(3+4 \lambda_{1}\right)}-1\right), \\
\Theta_{6}(t)=e^{-2 t H_{b} \lambda_{1}}\left(\Theta_{6}(0) e^{-3 t H_{b}}+A_{6}\left(e^{-3 t H_{b}}-1\right)\right)
\end{gathered}
$$

where $A_{4}$ and $A_{5}$ are arbitrary constants. One should note that $H_{a}-H_{b}=2 \lambda_{1} H_{b}$ and $H_{b}\left(3+4 \lambda_{1}\right)=2 H_{a}+H_{b}$.

The nontrivial, pseudoscalar mode, Einstein equations imply

$$
\begin{aligned}
& 0=\left(H_{b}\left(3+4 \lambda_{1}\right) \partial_{t}+\partial_{t}^{2}\right) \Psi_{2}(t), \\
& 0=\left(2 H_{b}^{2} \lambda_{1}\left(3+2 \lambda_{1}\right)+H_{b}\left(3+4 \lambda_{1}\right) \partial_{t}+\partial_{t}^{2}\right) \Psi_{3}(t),
\end{aligned}
$$

where we have again used the background solution, Eq. (27). The third equation vanishes identically, so there are no constraints on $\Psi_{1}(t)$.

The solutions for the other modes are

$$
\begin{aligned}
& \Psi_{2}(t)=\Psi_{2}(0) e^{-t H_{b}\left(3+4 \lambda_{1}\right)}+B_{2}\left(e^{-t H_{b}\left(3+4 \lambda_{1}\right)}-1\right), \\
& \Psi_{3}(t)=e^{-2 t H_{b} \lambda_{1}}\left(\Psi_{3}(0) e^{-3 t H_{b}}+B_{3}\left(e^{-3 t H_{b}}-1\right)\right),
\end{aligned}
$$

where $B_{2}$ and $B_{3}$ are arbitrary constants.

The unconstrained scalar and pseudoscalar modes, $\Theta_{2}(t), \Theta_{3}(t)$, and $\Psi_{1}(t)$, are, unsurprisingly, gauge artifacts. Importantly, $\Psi_{3}(t)$ is unchanged under any transformation of the form (25). We show this explicitly in the Appendix.

That $\Psi_{3}(t)$ is not a gauge artifact implies that $\beta_{1}$ (recalling that $\lambda_{1}=8 \pi G m^{2} \beta_{1}$ ) must be greater than zero,

$$
\beta_{1}>0 \text {, }
$$

in order for this mode to decay. Thus, assuming Eq. (56), no comoving modes grow in the limit $k / H \ll 1$.

For the timelike vector [10], the $\beta_{1}>0$ bound guarantees a positive definite flat-space Hamiltonian at second order in perturbations; however, $\beta_{1}>0$ is not a requirement for lowest order perturbative stability. For the case of a timelike vector in de Sitter space, all comoving modes decay as $1 / a(t)$ [10]; however, for a spacelike vector we find that $\Theta_{6}(t)$ and $\Psi_{3}(t)$ fall off more slowly than $1 / a(t)$ while $\Theta_{1}(t), \Theta_{4}(t), \Theta_{5}(t)$, and $\Psi_{2}(t)$ decay more quickly. (Here we assume that $H_{a}-H_{b}=2 H_{b} \lambda_{1}$ is smaller than $H_{a}$.)

\section{FLAT-SPACE ENERGIES}

In the previous sections we have studied the classical equations of motion linearized about the homogeneous background solution. For small $\mathrm{Gm}^{2}$ they were stable provided $\beta_{1}>0$ and $\beta_{1}+\beta_{2}+\beta_{3} \geq 0$. In this section we examine the classical energy of solutions to the flat- space equations of motion. Negative energy solutions indicate an instability that might not have shown up in our study of the linearized equations of motion.

In flat space one can write the vector field action as

$$
\begin{aligned}
S_{u}= & -\int d^{4} x\left(\frac{\beta_{1}}{2} F_{\mu \nu} F^{\mu \nu}+\left(\beta_{1}+\beta_{2}+\beta_{3}\right)\left(\partial_{\mu} u^{\mu}\right)^{2}\right. \\
& \left.-\lambda\left(u_{\mu} u^{\mu}-m^{2}\right)\right)
\end{aligned}
$$

where $F_{\mu \nu}=\partial_{\mu} u_{\nu}-\partial_{\nu} u_{\mu}$ and boundary terms are set to zero. Let $i, j \in\{1,2\}$ as in previous sections. The energy functional derived from the above action is ${ }^{5}$

$$
\begin{aligned}
E_{u}= & \int d^{3} x T_{0}^{(u) 0}=\int d^{3} x\left(\frac{\delta \mathcal{L}_{u}}{\delta\left(\partial_{0} u_{\rho}\right)} \partial_{0} u_{\rho}-\mathcal{L}_{u}\right) \\
= & \beta_{1} \int d^{3} x\left(\frac{1}{2} F_{\mu \nu} F_{\mu \nu}+2 u_{0} \partial_{\mu} F_{\mu 0}\right) \\
& -\left(\beta_{1}+\beta_{2}+\beta_{3}\right) \int d^{3} x\left(\left(\partial_{0} u_{0}\right)^{2}\right. \\
& \left.-\left(\partial_{i} u_{i}+\partial_{3} u_{3}\right)^{2}\right)-\int d^{3} x \lambda\left(u_{\mu} u^{\mu}-m^{2}\right),
\end{aligned}
$$

where, in the last expression, all indices are intentionally lower, and repeated indices should be summed without factors of the metric.

We take $u_{\mu}=\left(\boldsymbol{v}_{0}, \boldsymbol{v}_{1}, \boldsymbol{v}_{2}, m+\boldsymbol{v}_{3}\right)$ as in Sec. III. Physical field configurations must satisfy the Lagrange multiplier equation of motion, $u^{2}=m^{2}$, which implies

$$
v_{3}=m\left(\sqrt{1+\frac{v_{0}^{2}-v_{i}^{2}}{m^{2}}}-1\right)=\frac{v_{0}^{2}-v_{i}^{2}}{2 m}+\ldots
$$

Thus $v_{3}$ is actually second order in perturbations. This expansion also makes manifest that an expansion order by order in perturbations is equivalent to an expansion in powers of $m^{-1}$.

Imposing only the Lagrange multiplier equation of motion, the lowest order $\left[\mathcal{O}\left(m^{0}\right)\right]$ piece of Eq. (58) is

$$
\begin{aligned}
E_{u}^{(0)}= & \beta_{1} \int d^{3} x\left(\frac{1}{2} F_{i j} F_{i j}-\left(\partial_{3} v_{0}\right)^{2}+\left(\partial_{3} v_{i}\right)^{2}\right. \\
& \left.-\left(\partial_{i} v_{0}+\partial_{0} v_{i}\right) F_{i 0}\right)-\left(\beta_{1}+\beta_{2}+\beta_{3}\right) \\
& \times \int d^{3} x\left(\left(\partial_{0} v_{0}\right)^{2}-\left(\partial_{i} v_{i}\right)^{2}\right),
\end{aligned}
$$

since $v_{3}=\mathcal{O}\left(m^{-1}\right)$. When $\beta_{1}+\beta_{2}+\beta_{3} \neq 0$, we find that the mode described by the dispersion relation (21) has energy density (at lowest order in perturbations)

$$
E_{u}^{(0)} / V=-2 \beta_{1} \omega^{2} k_{3}^{2} \tilde{\theta}_{2}^{2},
$$

\footnotetext{
${ }^{5}$ The Lagrange multiplier constraint is holonomic. Whether a holonomic constraint is imposed before or after forming the stress-energy tensor has no effect on the tensor's final form.
} 
while the modes that propagate with the dispersion relation $\omega^{2}=k_{\perp}^{2}+k_{3}^{2}$ have energy density

$$
E_{u}^{(0)} / V=2 \beta_{1} \omega^{2} k_{\perp}^{2} \tilde{\psi}^{2} \quad \text { and } \quad E_{u}^{(0)} / V=2 \beta_{1} k_{3}^{2} k_{\perp}^{2} \tilde{\theta}_{2}^{2},
$$

where $\tilde{\psi}$ and $\tilde{\theta}_{2}$ are coordinate-independent coefficients of the plane wave solutions and $V$ is the volume of space. Thus for $\beta_{1}>0$ the mode with dispersion relationship (21) has negative energy while the others have positive energy. The existence of a negative energy mode implies that the background field configuration, $\bar{u}^{\mu}=m \eta^{\mu 3}$, is unstable when $\beta_{1}+\beta_{2}+\beta_{3} \neq 0$.

However, as discussed in Sec. III, when $\beta_{1}+\beta_{2}+$ $\beta_{3}=0$, the mode that we have just shown to have negative energy (when $\beta_{1}>0$ ) vanishes. In fact, we can show that in this case solutions to the Euler-Lagrange equations of motion that are near the background $\bar{u}^{\mu}=m \eta^{\mu 3}$ have non-negative energy. From here on, we take $\beta_{1}+\beta_{2}+$ $\beta_{3}=0$. First note that $\lambda=\mathcal{O}\left(\mathrm{m}^{-1}\right)$ since, by the equations of motion,

$$
\begin{aligned}
-\lambda / \beta_{1} & =\frac{u_{\nu} \partial_{\mu} F^{\mu \nu}}{m^{2}}=\frac{\partial_{\mu} F^{\mu 3}}{m}+\frac{v_{\nu} \partial_{\mu} F^{\mu \nu}}{m^{2}} \\
& =\frac{\partial_{0} \partial_{3} v_{0}-\partial_{i} \partial_{3} v_{i}}{m}+\mathcal{O}\left(m^{-2}\right) .
\end{aligned}
$$

Therefore, the equations of motion, $\beta_{1} \partial_{\mu} F^{\mu \nu}=-\lambda u^{\nu}$, imply $\partial_{\mu} F_{\mu 0}=\mathcal{O}\left(\mathrm{m}^{-1}\right)$. This implies that field configurations that satisfy the equations of motion have the lowest order energy functional,

$E_{u}^{(0)}=\beta_{1} \int d^{3} x\left(F_{0 i} F_{0 i}+\frac{1}{2} F_{i j} F_{i j}+\left(\partial_{3} v_{0}\right)^{2}+\left(\partial_{3} v_{i}\right)^{2}\right)$.

Thus the energy functional is positive semidefinite at lowest order in perturbations.

In principle, there could be a direction in field space that has zero energy at lowest order but has a negative energy contribution at a higher order in perturbations that leads to an instability. Thus let us consider field configurations that have zero energy at lowest order,

$$
F_{0 i}=F_{i j}=\partial_{3} v_{0}=\partial_{3} v_{i}=0 .
$$

Recall that the full energy functional (assuming $u_{\mu} u^{\mu}=$ $\left.m^{2}\right)$ is

$$
\begin{aligned}
E_{u}= & \beta_{1} \int d^{3} x\left(F_{0 i} F_{0 i}+F_{03} F_{03}+\frac{1}{2} F_{i j} F_{i j}+F_{i 3} F_{i 3}\right. \\
& \left.+2\left(\partial_{i} F_{i 0}+\partial_{3} F_{30}\right) u_{0}\right) .
\end{aligned}
$$

For field configurations that have zero energy at leading order-those that satisfy (62) - the full energy functional is

$$
E_{u}=\beta_{1} \int d^{3} x\left(\left(\partial_{0} v_{3}\right)^{2}+\left(\partial_{i} v_{3}\right)^{2}\right),
$$

where $v_{3}=m\left(\sqrt{1-\left(\boldsymbol{v}_{i}^{2}-\boldsymbol{v}_{0}^{2}\right) / m^{2}}-1\right)$. Thus, if $\boldsymbol{v}_{3}$ is not a constant, then the energy given in Eq. (64) is strictly positive.

The energies of field configurations about the background $\bar{u}^{\mu}=m \eta^{\mu 3}$ that could possibly have turned negative locally - those that have zero energy at leading order-are in fact positive semidefinite to all orders [as we have shown in Eq. (64)]. In Ref. [18], the energy in this theory was found to be unbounded from below, but the existence of local extrema was not considered. Our analysis suggests that the background about which we expand is metastable.

\section{CONCLUDING REMARKS}

We have studied the small fluctuations about a spatially homogeneous anisotropic inflationary background. The anisotropy was caused by a dynamical four-vector that was constrained to have a spacelike invariant norm. For a range of parameters the background space-time is classically stable.

From the first order stability analysis of the equations of motion, we derived the constraints $\beta_{1}>0$ and $\beta_{1}+\beta_{2}+$ $\beta_{3} \geq 0$ for the parameters in the vector Lagrangian (1). Moreover, we find that all modes have positive energy in flat space and do not grow with time in the case where the kinetic term for the four-vector corresponds to a field strength tensor squared (i.e. for $\beta_{1}+\beta_{2}+\beta_{3}=0$ ). A negative energy mode propagates if $\beta_{1}+\beta_{2}+\beta_{3} \neq 0$, which implies that the background given by Eqs. (2)-(5) is unstable when $\beta_{1}+\beta_{2}+\beta_{3} \neq 0$. The flat-space energy is greater than or equal to its value at the background $\bar{u}^{\mu}=$ $m \eta^{\mu 3}$ when $\beta_{1}>0$ and $\beta_{1}+\beta_{2}+\beta_{3}=0$, which suggests that the background is metastable in this case. ${ }^{6}$ At the classical level the models of Refs. [7,9,17] with a spacelike four-vector and $\beta_{1}+\beta_{2}+\beta_{3}=0$ provide a convenient framework to explore the consequences of a small violation of rotational invariance during the inflationary era.

\section{ACKNOWLEDGMENTS}

We thank Sean Carroll, Ted Jacobson, and Lotty Ackerman for their comments during the preparation of this manuscript. This work was supported in part by DOE Grant No. DE-FG03-92ER40701.

\section{APPENDIX: GAUGE ARTIFACTS}

A gauge transformation of the form in Eq. (25) induces the transformation $h_{\mu \nu}(t, \vec{k}) \rightarrow h_{\mu \nu}(t, \vec{k})+\Delta h_{\mu \nu}(t, \vec{k})$ in the comoving Fourier-transformed metric perturbations. Explicitly $(i, j=1,2)$,

\footnotetext{
${ }^{6} \mathrm{An}$ analysis of energies when gravity is included is likely to change the condition from $\beta_{1}+\beta_{2}+\beta_{3}=0$ to $\beta_{1}=-\beta_{3}$, $\beta_{2}=0$.
} 
$\Delta h_{00}(t, \vec{k})=2 \partial_{t} f_{0}\left(t, \vec{k}_{\perp}\right)$,

$\Delta h_{0 i}(t, \vec{k})=i \frac{k_{i}}{a(t)} f_{0}\left(t, \vec{k}_{\perp}\right)-a(t) \partial_{t} f_{i}\left(t, \vec{k}_{\perp}\right)$,

$\Delta h_{03}(t, \vec{k})=b(t)\left(i \delta^{\prime}\left(k_{3}\right) \partial_{t} f_{0}\left(t, \vec{k}_{\perp}\right)-\partial_{t} f_{3}\left(t, \vec{k}_{\perp}\right)\right)$,

$\Delta h_{i j}(t, \vec{k})=-2\left(H_{a} \delta_{i j} f_{0}\left(t, \vec{k}_{\perp}\right)+i k_{(i} f_{j)}\left(t, \vec{k}_{\perp}\right)\right)$,

$\Delta h_{i 3}(t, \vec{k})=-\frac{b(t)}{a(t)} k_{i}\left(\delta^{\prime}\left(k_{3}\right) f_{0}\left(t, \vec{k}_{\perp}\right)+i f_{3}\left(t, \vec{k}_{\perp}\right)\right)$,

where $\vec{k}_{\perp}=\left(k_{1}, k_{2}\right)$ and $f_{\mu}\left(t, \vec{k}_{\perp}\right)$ are the Fourier transforms of $f_{\mu}\left(t, x_{1}, x_{2}\right)$. The corresponding transformations of the fields as defined in Eq. (49) are

$$
\begin{gathered}
\Delta \Theta_{1}=\Delta h_{00}=2 \partial_{t} f_{0}\left(t, \vec{k}_{\perp}\right), \\
\Delta \Theta_{2}=\frac{k_{i}}{k_{\perp}} \Delta h_{0 i}=i \frac{k_{\perp}}{a(t)} f_{0}\left(t, \vec{k}_{\perp}\right)-a(t) \frac{k_{i}}{k_{\perp}} \partial_{t} f_{i}\left(t, \vec{k}_{\perp}\right), \\
\Delta \Theta_{3}=\Delta h_{03}=b(t)\left(i \delta^{\prime}\left(k_{3}\right) \partial_{t} f_{0}\left(t, \vec{k}_{\perp}\right)-\partial_{t} f_{3}\left(t, \vec{k}_{\perp}\right)\right), \\
\Delta \Theta_{4}=\Delta h_{i i}=-2\left(H_{a} 2 f_{0}\left(t, \vec{k}_{\perp}\right)+i k_{i} f_{i}\left(t, \vec{k}_{\perp}\right)\right), \\
\Delta \Theta_{5}=\left(\frac{k_{i} k_{j}}{k_{\perp}^{2}}-\frac{1}{2} \delta_{i j}\right) \Delta h_{i j}=-i k_{i} f_{i}\left(t, \vec{k}_{\perp}\right),
\end{gathered}
$$

$$
\begin{aligned}
\Delta \Theta_{6} & =\frac{k_{i}}{k_{\perp}} \Delta h_{i 3} \\
& =-\frac{b(t)}{a(t)} k_{\perp}\left(\delta^{\prime}\left(k_{3}\right) f_{0}\left(t, \vec{k}_{\perp}\right)+i f_{3}\left(t, \vec{k}_{\perp}\right)\right),
\end{aligned}
$$

and

$$
\begin{gathered}
\Delta \Psi_{1}=\frac{\epsilon_{i j} k_{j}}{k_{\perp}} \Delta h_{0 i}=-a(t) \frac{\epsilon_{i j} k_{j}}{k_{\perp}} \partial_{t} f_{i}\left(t, \vec{k}_{\perp}\right), \\
\Delta \Psi_{2}=\frac{k_{(i} \epsilon_{j) k} k_{k}}{k_{\perp}^{2}} \Delta h_{i j}=i \epsilon_{i j} k_{i} f_{j}\left(t, \vec{k}_{\perp}\right), \\
\Delta \Psi_{3}=\frac{\epsilon_{i j} k_{j}}{k_{\perp}} \Delta h_{i 3}=0 .
\end{gathered}
$$

We see that $\Psi_{3}(t)$ is invariant under the residual gauge transformations in Eq. (25). From Eqs. (A2), (A3), and (A7), it is clear that one can gauge away $\Theta_{3}(t)$ by fixing $\partial_{t} f_{3}\left(t, \vec{k}_{\perp}\right)$ and $\partial_{t} f_{0}\left(t, \vec{k}_{\perp}\right)=0$, and one can gauge away $\Theta_{2}(t)$ and $\Psi_{1}(t)$ by fixing $\partial_{t} k_{i} f_{i}\left(t, \vec{k}_{\perp}\right)$ and $\partial_{t} \epsilon_{i j} k_{j} f_{i}\left(t, \vec{k}_{\perp}\right)$, respectively.
[1] L. Ackerman, S. M. Carroll, and M. B. Wise, Phys. Rev. D 75, 083502 (2007).

[2] A. E. Gumrukcuoglu, C. R. Contaldi, and M. Peloso, J. Cosmol. Astropart. Phys. 11 (2007) 005.

[3] A. E. Gumrukcuoglu, C. R. Contaldi, and M. Peloso, arXiv:astro-ph/0608405.

[4] A. R. Pullen and M. Kamionkowski, Phys. Rev. D 76, 103529 (2007).

[5] S. Ando and M. Kamionkowski, Phys. Rev. Lett. 100, 071301 (2008).

[6] C. G. Boehmer and D. F. Mota, arXiv:0710.2003.

[7] V. A. Kostelecky and S. Samuel, Phys. Rev. D 40, 1886 (1989).

[8] T. Jacobson and D. Mattingly, Phys. Rev. D 70, 024003 (2004).

[9] S. M. Carroll and E. A. Lim, Phys. Rev. D 70, 123525 (2004).

[10] E. A. Lim, Phys. Rev. D 71, 063504 (2005).
[11] C. Eling and T. Jacobson, Phys. Rev. D 69, 064005 (2004).

[12] B.Z. Foster and T. Jacobson, Phys. Rev. D 73, 064015 (2006).

[13] B. Li, D. Fonseca Mota, and J. D. Barrow, Phys. Rev. D 77, 024032 (2008).

[14] M. D. Seifert, Phys. Rev. D 76, 064002 (2007).

[15] B.Z. Foster, Phys. Rev. D 73, 104012 (2006).

[16] C. Eling and T. Jacobson, Classical Quantum Gravity 23, 5625 (2006).

[17] T. Jacobson and D. Mattingly, Phys. Rev. D 64, 024028 (2001).

[18] M. A. Clayton, arXiv:gr-qc/0104103.

[19] R. Bluhm, S.-H. Fung, and V. A. Kostelecky, Phys. Rev. D 77, 065020 (2008).

[20] T. Jacobson, arXiv:0801.1547.

[21] T.S. Pereira, C. Pitrou, and J.-P. Uzan, J. Cosmol. Astropart. Phys. 09 (2007) 006.

[22] K. Tomita and M. Den, Phys. Rev. D 34, 3570 (1986). 